\title{
Evaluación de un arreglo de diodos en 2 dimensiones para dosimetría física y clínica de rutina en haces de electrones en radioterapia.
}

\author{
María José SÁnchez-Lovell ${ }^{1}$ and IsaAC RoJas-Campos ${ }^{2}$ \\ ${ }^{1}$ Hospital México, mail: mjslq@yahoo.com \\ ${ }^{2}$ Centro Médico de Radioterapia Irazú, mail: isaac.rojas@radioterapiairazu.net
}

Recibido: 7 de Abril de 2015 / Aceptado: 4 de Mayo de 2015

\begin{abstract}
Resumen
In this study, we intend to show the feasibility of using a 2-dimensional diode array for routinely electron dosimetry. We calibrated the array with energies 9, 12 and $16 \mathrm{MeV}$, and evaluated the central beam axis dose, flatness, symmetry and short term reproducibility against measurements with parallel-plane ionization chamber. We used gamma analysis for comparison of exported and measured fluencies. In short term reproducibility the variation coefficient was less than $1.0 \%$ for all the energies. The absolute difference in flatness and symmetry was less than 3.0\%. In the gamma analysis, the percentages of the diode array data points that match with the planned dose were: $90.8 \%$ to $97.9 \%$ for $9 \mathrm{MeV}, 52.7 \%$ to $81.7 \%$ for $12 \mathrm{MeV}$ and $69.2 \%$ to $94.2 \%$ for $16 \mathrm{MeV}$. The absolute differences between the planned and measured dose were less than to $2.0 \%$, except for $12 \mathrm{MeV}$ (3.2\%). The agreement between the data obtained with ionization chamber and the diode array supports the use of Mapcheck as a trustable method for electron dosimetry.
\end{abstract}

Keywords: Clinical electron dosimetry, diode array, Mapcheck

\begin{abstract}
En este estudio, se tiene la intención de mostrar la factibilidad de utilizar un arreglo de diodos bidimensional para dosimetría de electrones. Se calibró el arreglo con energías de 9, 12 y $16 \mathrm{MeV}$. Se evaluaron la dosis en eje central, planicidad, simetría, reproducilbilidad a corto plazo, contra mediciones con cámara de ionización plano-paralela y se realizó gamma análisis para la comparación de mapas de fluencias exportadas y medidas. En reproducibilidad a corto plazo se obtuvieron coeficientes de variación menores al $1.0 \%$ para todas las energías. Para planicidad y simetría se obtuvieron diferencias absolutas menores a 3.0\%. En el gamma análisis, los porcentajes de los puntos de datos del arreglo de diodos que concordaron con la dosis planificada fueron: 90.8 a $97.9 \%$ para $9 \mathrm{MeV}$, de 52.7 a $81.7 \%$ para 12 $\mathrm{MeV}$ y de 69.2 a $94.2 \%$ para $16 \mathrm{MeV}$. Las diferencias en dosis absoluta entre el plan y la medición con Mapcheck fueron menores al $2.0 \%$, excepto para la energía de $12 \mathrm{MeV}$ (3.2\%). La concordancia entre los datos obtenidos con cámara de ionización y el arreglo de diodos respaldan el uso del Mapcheck como un método confiable para dosimetría de electrones.
\end{abstract}

Palabras clave: Dosimetría clínica de electrones, arreglo de diodos en dos dimensiones, Mapcheck.

\section{INTRODUCCIÓN}

$\mathrm{T}$ Radicionalmente, la caracterización de los haces de radiación se ha realizado con cámaras de ionización, que han sido por mucho tiempo el detector de referencia para dosimetría de fotones y electrones. [1] [6] Dicha metodología, que implica mediciones en agua, suele consumir mucho tiempo y es poco práctica cuando se desean realizar verificaciones de rutina, como en el caso de los planes de tratamiento.

Existe una alternativa a las cámaras de ionización para obtener datos de dosis rutinarios sin la necesidad de realizar mediciones en agua: los diodos semiconductores. El empleo de detectores semiconductores se ha incrementado en la práctica de radioterapia para dosimetría de campos pequeños y para dosimetría in vivo. Las principales ventajas de estos dispositivos son: su dimensión y su gran sensibilidad a radiación ionizante. Han sido ampliamente utilizados cuando se requiere alta resolución espacial debido a su pequeño volumen sensible. Adicionalmente, se tiene la ventaja de que si se cuenta con un arreglo de diodos se pueden realizar mediciones en varios puntos con una sola irradiación. [6]

Desde el año 1970, se han utilizado para uso clínico haces de electrones de alta energía con energías entre 4 y 25 $\mathrm{MeV}$. En tratamientos con electrones, la ausencia de verificación es un tema delicado, ya que esta terapia aún se administra en muchos centros de países en desarrollo con base en consideraciones clínicas, sin una planificación de tratamiento formal.15. Además, en el caso particular de electrones, cuando las dimensiones que definen el área del campo son menores que el rango práctico de penetración para la energía en cuestión, se tienen diferencias significativas en la dosimetría respecto a los campos de referencia; 
esto podría implicar que al aplicar un tratamiento con campo bloqueado, el haz entregado difiera del haz planificado, como consecuencia de la pérdida de equilibrio electrónico lateral. 9]

Adicionalmente, como parte importante del control de calidad del haz de radiación se encuentra el contar con métodos de medición independientes para verificar la dosimetría del haz y la dosis calculada por el sistema de planificación. Se debe tener especial cuidado cuando se comparan las dosis medidas y planificadas. Si la dosis planificada es modelada con una cámara de ionización, las zonas de gradiente pueden subestimarse debido al efecto de dosis media volumétrica (conocido en inglés como "volumen averaging", que es el promedio sobre la función de respuesta espacial del detector) [15. Así mismo, en el control de calidad del haz de radiación con cámaras de ionización se subestiman los valores cuando hay alto gradiente de dosis. Complementar el control de calidad con un arreglo de diodos puede resultar favorable para revelar inconsistencias entre la dosis planificada y la dosis entregada, que no saltarían a la vista si la verificación del plan se realiza con el mismo equipo que se utilizó para modelar el acelerador lineal en el sistema de planificación.

Los arreglos de diodos en dos dimensiones como el Mapcheck, fabricado por Sunnuclear, son muy utilizados en la actualidad para verificación de los planes de tratamiento para IMRT con fotones, debido a que permiten un gran número de mediciones puntuales en el plano del haz, con poco tiempo de instalación del equipo y medición, y con una resolución adecuada (menor que la de dosimetría de film, pero aceptable para fines de control de calidad). El empleo de estos dispositivos en la verificación de tratamientos para obtener distribuciones de dosis brinda mucha más información que el método tradicional de realizar una medición de dosis puntual con cámara de ionización. [1] [16]

Múltiples artículos han descrito la fiabilidad de los diodos semiconductores para controles de calidad de técnicas avanzadas de tratamiento, [1] 3] [10] [4 pero pocos describen la factibilidad de emplear estos detectores en dosimetría de electrones. 6] [5] Khoury, $\mathrm{H}$ et al 6] , realizaron la evaluación de un diodo de Si comercial para su uso en dosimetría de electrones y encontraron una relación lineal de la respuesta con la dosis, así como buena concordancia entre los perfiles de dosis en profundidad obtenidos con diodos contra los obtenidos con cámara de ionización .Guo, F et al [5] mostraron resultados preliminares que demuestran que el Mapcheck puede ser utilizado para realizar mapas de comparación de dosis para electrones de forma rápida y relativamente aceptable. Watts 14 demostró la utilidad de un arreglo de diodos para medición de planicidad y simetría en haces de electrones, y para detectar cambios en la energía del haz.

El objetivo de este estudio es evaluar la factibilidad de utilizar el Mapcheck para dosimetría de electrones de 9,
12 y $16 \mathrm{MeV}$, para verificación de campos de tratamientos.

\section{MÉTODOS}

\section{A. Arreglo de diodos Mapcheck Sunnuclear.}

El Mapcheck consiste en un arreglo de diodos en dos dimensiones. Presenta un grosor físico de $1.35 \mathrm{~cm}$ (o 2 $\mathrm{g} / \mathrm{cm}^{2}$ agua-equivalente), [1] [3] [4] y un material para retrodispersión equivalente a $2.3 \mathrm{~cm}$ de agua. Este material dispersor inherente reemplaza el blindaje usual alrededor de los diodos para disminuir su respuesta a fotones de rayos $\mathrm{X}$ dispersados de baja energía. 4] Contiene en total 445 diodos de silicio ( $\mathrm{Si}$ ), en una conformación octogonal de $22 \mathrm{~cm}$. La región central de $10 \times 10 \mathrm{~cm}^{2}$ contiene diodos espaciados $7 \mathrm{~mm}$ entre sí. En el área externa que rodea a la grilla central, los diodos están espaciados $14 \mathrm{~mm}$. Durante la irradiación, los detectores generan una carga proporcional a la dosis recibida en esa locación. Cada diodo está conectado a un amplificador operacional MOSFET que integra la carga. Posteriormente, la carga es convertida de una señal análoga a una señal digital y enviada a una computadora personal (conectada a través de un circuito interfase amplificador) que corrige cada dato con el factor de corrección para cada locación que está almacenada en la memoria. De esta forma se obtiene la dosis absoluta en cada punto de medición. [1] 3] [10] [4] [13]

Los diodos del Mapcheck son de Si tipo - N (sustrato dopado con fósforo), [1] con un build up intrínseco menor a $0.1 \mathrm{gm} / \mathrm{cm}^{2}$, y están especialmente diseñados para mediciones en la superficie del paciente. 3. La densidad electrónica del Si es 18000 veces mayor que la del aire, por lo que un diodo con un volumen mucho menor al de una cámara de ionización puede tener una sensibilidad 10 veces mayor. 13 Estos diodos presentan respuesta lineal con la dosis hasta una dosis acumulada máxima dependiente de la energía (450 cGy para $9 \mathrm{MeV}$, según medición) debido al tamaño del capacitor empleado en el circuito integrador. 4. Por encima de esta dosis, hay saturación en la respuesta. [3] Los diodos fabricados por Sunnuclear son muy resistentes a daño por radiación (la degradación por radiación es insignificante a corto y a largo plazo) debido a características particulares de su fabricación. [1] [4]

A diferencia de las cámaras de ionización, los arreglos de diodos no requieren un tiempo de calentamiento o aplicación de voltaje previo a su uso, disminuyendo así el tiempo requerido para las mediciones. [13]

Los diodos se encuentran posicionados entre dos platos acrílicos con superficies conductoras montadas en una tarjeta de circuito multicapas. Esto permite blindar los campos de radiofrecuencia generados por el acelerador lineal. [10]

El Mapcheck tiene una resolución aceptable $(0.8 \times 0.8$ 
mm) haciéndolo ideal para dosimetría de electrones con campos pequeños y para análisis en regiones con alto gradientes de dosis. [3] [4]

La respuesta de los diodos presenta dependencia con la temperatura pero no con la presión (a diferencia de la lectura de las cámaras de ionización, que deben corregirse por ambos factores de influencia).

La dependencia con la energía es fácilmente manejada con un archivo de calibración para cada energía. Tanto la calibración como las mediciones con el Mapcheck se realizan con el eje central del haz perpendicular al plano de los diodos, por lo que no se requieren correcciones por ángulo de incidencia. Adicionalmente, las mediciones de dosis se deben realizar bajo las condiciones de referencia empleadas en la calibración. [15] [10] [4] 13]

Guo $\mathrm{F}$ et al 5] no recomiendan el uso del arreglo de diodos con energías menores a $9 \mathrm{MeV}(\mathrm{d}$ máx $=2 \mathrm{~cm})$, debido al build up inherente al equipo, por lo que no se utiliza la energía de $6 \mathrm{MeV}$ para el estudio.

\section{B. Calibración del arreglo de diodos y toma de datos con el sistema CRS.}

El CRS es un sistema de barrido con cámara de ionización en tres dimensiones, apto para realizar perfiles de campo en ejes "X", "Y", diagonales y perfiles de dosis en profundidad. (eje Z) Con una cámara de ionización Exradin Ion Chamber A1SL (primaria) y el sistema de barrido CRS se realizaron perfiles de dosis en profundidad y perfiles de dosis de campo en los ejes "X" (transversal respecto a la camilla) e "Y" (longitudinal respecto a la camilla) para las energías de estudio, con conos de $6 \times 6$ $\mathrm{cm}^{2}, 10 \times 10 \mathrm{~cm}^{2}$ y $15 \times 15 \mathrm{~cm}^{2}$ a profundidad de $2 \mathrm{~cm}$. Se determinaron los valores de planicidad y simetría a partir de los perfiles de campo. Dichos términos y método de cálculo se encuentran en el reporte número 13 de la AAPM. 2

Se obtuvieron valores de dosis absoluta con cámara de ionización plano paralela PTW Markus 23343, en fantoma de agua según el protocolo TRS-398. [8] Se utilizaron las curvas de perfil de dosis en profundidad para obtener los valores de dosis absoluta en el eje central (CAX) a $2 \mathrm{~cm}$, y se introdujeron dichos valores en el programa de cómputo del Mapcheck para la calibración de los diodos en dosis absoluta.

El proceso de calibración del arreglo de diodos se realizó con un cono de $25 \times 25 \mathrm{~cm}^{2}$, SSD de $100 \mathrm{~cm}$, y build up de $2 \mathrm{~g} / \mathrm{cm}^{2}$ (build up inherente). Para la calibración de dosis en el eje central se entregó una dosis conocida en un campo de $10 \times 10 \mathrm{~cm}^{2}$, con el equipo bajo las condiciones de referencia indicadas previamente. [15] 12] Se irradió con 100 UM, y se introdujeron los valores de dosis absoluta en el equipo, para que el software pudiera relacionar la señal con la dosis entregada. 12

Se determinó la reproducibilidad a corto plazo a partir de 15 medidas consecutivas de $60 \mathrm{UM}$, con los diodos del Mapcheck posicionados a SSD de $100 \mathrm{~cm}$ y profundidad de $2 \mathrm{~g} / \mathrm{cm}^{2}$.

Xio es un sistema de planificación, que utiliza el algoritmo Montecarlo (eMC) para el cálculo de dosis con haces de electrones. Los sistemas de planificación como Xio utilizan aproximaciones que pueden disminuir el tiempo de cálculo pero sacrifican la exactitud. 11] Se crearon planes de tratamiento con el sistema de planificación XIO para las energías de estudios con un fantoma virtual, con el punto de verificación a $2 \mathrm{~cm}$ de profundidad. Se exportaron las fluencias en formato compatible con el mapcheck (.txt) y se midieron con el arreglo de diodos. Se irradió el arreglo según los plantes de tratamiento creados, con un Varian Clinac 1800. Los planes exportados se compararon contra los mapas de dosis medidos, utilizando un criterio de comparación de $\pm 3 \%$ de diferencia de dosis y \pm 3 $\mathrm{mm}$ de distancia para concordancia, dentro de un umbral de isodosis del $10 \%$. La configuración de haces fue de $180^{\circ}$, con tamaños de cono de $6 \times 6 \mathrm{~cm}^{2}, 10 \times 10 \mathrm{~cm}^{2}$ y $15 \times 15 \mathrm{~cm}^{2}$ para las distintas energías.

El cuadro 1, es un comparativo entre las carácterísticas del Mapcheck, la cámara de ionización plano paralela y la cámara utilizada con el CRS para los barridos.

\section{RESULTADOS}

En este estudio evaluamos la capacidad de un arreglo de diodos en dos dimensiones para ser utilizado en control de calidad dosimétrico y control de calidad del sistema de planificación; ésto con el fin de incorporar en la rutina diaria de la clínica el control de calidad de los planes de tratamiento de electrones.

Los resultados obtenidos para la reproducibilidad a corto plazo con el arreglo de diodos fueron $0.2 \%$ para 9 $\mathrm{MeV}, 0.1 \%$ para $12 \mathrm{MeV}$ y $0.2 \%$ para $16 \mathrm{MeV}$. Debe recordarse que este valor incluye no solo la reproducibilidad del diodo, sino también la reproducibilidad del "beam output" entre medidas.

En los cuadros 2, 3 y 4 se muestran los resultados de planicidad y simetría obtenidos con la cámara y el arreglo bajo las condiciones de referencia para diferentes tamaños de campo.

Para la energía de $9 \mathrm{MeV}$ se obtuvieron diferencias absolutas en planicidad entre $-0.3 \%$ y $-1.7 \%$ entre las fluencias medidas con CRS y Mapcheck. Para $12 \mathrm{MeV}$ el rango de diferencias absolutas en planicidad en ambos ejes está entre $0.0 \%$ y $-1.4 \%$, y para la energía de $16 \mathrm{MeV}$ las diferencias absolutas están dentro del rango de $0.1 \%$ a $-1.0 \%$. 


\begin{tabular}{lllr}
\hline & Mapcheck Sunnuclear & PTW Markus 23343 & Exradin A1SL \\
\hline Dimensión activa $(\mathrm{mm})$ & Largo $\times$ ancho: $0.80 \times 0.80$ & 2.65 (radio) & 2.00 (radio) \\
Área activa $\left(\mathrm{mm}^{2}\right)$ & 0.64 & 22.00 & 13.00 \\
Grosor sensible $(\mathrm{mm})$ & 0.03 & 2.00 & - \\
Volumen sensible $\left(\mathrm{cm}^{3}\right)$ & $1.90 \times 10^{-5}$ & $5.50 \times 10^{-2}$ & $5.30 \times 10^{-2}$ \\
Sensibilidad $(\mathrm{nC} / \mathrm{Gy})$ & 32.00 & 2.00 & - \\
\hline
\end{tabular}

Cuadro 1: Datos de dimensión, área, volumen y sensibilidad del Mapcheck, la cámara de ionización plano paralela PTW Markus 23343 y la cámara A1SL.*

\begin{tabular}{llllllr}
\hline \multicolumn{1}{c}{ CRS } & Mapcheck & Dif.abs. & CRS & Mapcheck & Dif. abs. \\
\hline En X & Planicidad (\%) & Planicidad (\%) & $\%$ & Simetría (\%) & Simetría (\%) & $\%$ \\
$10 \times 10$ & 4.3 & 2.5 & -1.7 & 0.0 & 0.4 & 0.4 \\
$15 \times 15$ & 2.0 & 1.5 & -0.6 & 0.0 & 0.5 & 0.5 \\
En Y & Planicidad(\%) & Planicidad(\%) & $\%$ & Simetría(\%) & Simetría(\%) & $\%$ \\
$10 \times 10$ & 4.6 & 3.7 & -0.9 & 0.1 & 3.1 & 3.0 \\
$15 \times 15$ & 2.7 & 2.4 & -0.3 & 0.5 & 0.5 & 0.0 \\
\hline
\end{tabular}

Cuadro 2: Datos de planicidad y simetría para la energía de $9 \mathrm{MeV}$

En lo que respecta a simetría, las diferencias absolutas entre CRS y Mapcheck están dentro del rango de $0.0 \%$ a $3.0 \%$ para $9 \mathrm{MeV}$; de $-0.1 \%$ y $1.0 \%$ para $12 \mathrm{MeV}$ y de $-0.5 \%$ a $1.5 \%$ para $16 \mathrm{MeV}$.

En el cuadro 5 se pueden observar los resultados del gamma análisis para las distintas energías. El porcentaje de los puntos que pasan estuvo dentro del rango de 52.7 \% hasta $97.9 \%$. La diferencia entre los puntos fue más importante para campos pequeños de $6 \times 6 \mathrm{~cm}^{2}$ y para la energía de $12 \mathrm{MeV}$.

En el cuadro 6 se muestran los porcentajes de error entre la dosis en el eje central derivada del comisionamiento del sistema de planificación TPS, y la dosis absoluta medida con cámara de ionización utilizada para la calibración del Mapcheck (C-B/C). El error relativo máximo fue de 1.4 $\%$ para la energía de $12 \mathrm{MeV}$.

Los datos de diferencia de dosis en el eje central de los planes exportados del sistema de planificación contra las mediciones con el arreglo de diodos, se muestran en el cuadro 7. Los porcentajes de diferencia se encuentran en su mayoría, por debajo del $2.0 \%$. Para las energías de 12 y $16 \mathrm{MeV}$, con cono de $6 \times 6 \mathrm{~cm}^{2}$, se tiene un valor de error ligeramente aumentado.

En las Figuras 1 y 2 se muestra la pantalla de visualización de dosis del software del Mapcheck. En estas se puede ver la distribución de dosis en un plano (el software también permite ver la distribución de dosis en 3 dimensiones) de la fluencia medida (set 1), de la fluencia importada del sistema de planificación (set 2), y el mapa de diferencia en la distribución de dosis entre ambos sets (set 1 - set 2 ), que permite ubicar en el plano, las zonas calientes y frías. En el cuadro inferior derecho se muestran los perfiles en $\mathrm{X}$ o Y de los sets de datos $1 \mathrm{y}$ 2. En el cuadrante del extremo izquierdo se muestran los resultados del análisis gama y de la comparación de dosis absoluta.

En las figuras 3(a) y 3(b) se muestran los perfiles de dosis para un campo de $15 \times 15 \mathrm{~cm}^{2}$ en el eje "Y" longitudinal, para energías 9 y $12 \mathrm{MeV}$ respectivamente. En ambos casos se obtuvo una buena concordancia entre la planicidad y simetría medida con cámara de ionización y con el Mapcheck.

\section{Discusión}

La profundidad seleccionada de $2 \mathrm{~cm}$ para las mediciones, responde a un hecho de practicidad en el posicionamiento del arreglo de diodos tanto para la calibración de cada energía como para la medición de los planes de tratamiento, tomando en cuenta el build up intrínseco del equipo. Esta profundidad era la que anteriormente recomendaba el IAEA con el protocolo el TRS-277 para energías nominales mayores o iguales a $10 \mathrm{MeV}$ y menores a $20 \mathrm{MeV}$. Con este protocolo se aseguraba una concordancia dentro del $1 \%$ con determinaciones basadas en métodos no ionométricos para todas las energías de electrones. 7 ]

La modificación en el protocolo e introducción a la nueva profundidad de referencia que recomienda el protocolo TRS-398 responde a que demostró reducir significativamente las variaciones entre máquinas y los factores de calibración de las cámaras, y su uso se justifica particularmente para cámaras plano-paralelas; 8 no implica que dichas condiciones sean exigidas o tengan mayor exactitud para mediciones con diodos. En nuestro caso particular, 


\begin{tabular}{llllllr}
\hline \multicolumn{1}{c}{ CRS } & Mapcheck & Dif.abs. & CRS & Mapcheck & Dif. abs. \\
\hline En X & Planicidad (\%) & Planicidad (\%) & $\%$ & Simetría (\%) & Simetría (\%) & $\%$ \\
$10 \times 10$ & 2.5 & 1.6 & -0.9 & 0.3 & 0.3 & 0.0 \\
$15 \times 15$ & 1.8 & 1.4 & -0.3 & 1.0 & 1.5 & 0.6 \\
En Y & Planicidad(\%) & Planicidad(\%) & $\%$ & Simetría(\%) & Simetría(\%) & $\%$ \\
$10 \times 10$ & 4.1 & 2.8 & -1.4 & 1.1 & 1.0 & -0.1 \\
$15 \times 15$ & 2.4 & 2.5 & 0.0 & 0.2 & 1.3 & 1.0 \\
\hline
\end{tabular}

Cuadro 3: Datos de planicidad y simetría para la energía de $12 \mathrm{MeV}$

\begin{tabular}{llllllr}
\hline & CRS & Mapcheck & Dif.abs. & CRS & Mapcheck & Dif. abs. \\
\hline En X & Planicidad (\%) & Planicidad (\%) & $\%$ & Simetría (\%) & Simetría (\%) & $\%$ \\
$10 \times 10$ & 2.0 & 1.3 & -0.7 & 0.3 & 0.2 & -0.1 \\
$15 \times 15$ & 1.4 & 1.3 & -0.1 & 0.8 & 1.6 & 0.9 \\
En Y & Planicidad(\%) & Planicidad(\%) & $\%$ & Simetría(\%) & Simetría(\%) & $\%$ \\
$10 \times 10$ & 3.9 & 2.8 & -1.0 & 1.5 & 1.0 & -0.5 \\
$15 \times 15$ & 2.5 & 2.6 & 0.1 & 0.9 & 2.4 & 1.5 \\
\hline
\end{tabular}

Cuadro 4: Datos de planicidad y simetría para la energía de $16 \mathrm{MeV}$

\begin{tabular}{lcllll}
\hline & \multicolumn{5}{c}{ Gamma análisis } \\
\hline Energía $(\mathrm{MeV})$ & Campo $\left(\mathrm{cm}^{2}\right)$ & Puntos totales & Pasan & Fallan & $\%$ Pasan \\
\hline 9 & $6 \times 6$ & 109 & 99 & 10 & 90.8 \\
& $10 \times 10$ & 241 & 236 & 5 & 97.9 \\
& $15 \times 15$ & 333 & 314 & 19 & 94.3 \\
12 & $6 \times 6$ & 129 & 68 & 61 & 52.7 \\
& $10 \times 10$ & 226 & 183 & 43 & 81.0 \\
16 & $15 \times 15$ & 333 & 272 & 61 & 81.7 \\
& $6 \times 6$ & 130 & 90 & 40 & 69.2 \\
& $10 \times 10$ & 226 & 213 & 13 & 94.2 \\
& $15 \times 15$ & 335 & 295 & 40 & 88.1 \\
\hline
\end{tabular}

Cuadro 5: Comparación de fluencias de electrones planificadas contra las fluencias medidas con el Mapcheck

puesto que el Mapcheck se calibra directamente con cada máquina, el factor de variabilidad entre máquinas no afectaría para fines del estudio.

Como se indica en los resultados, los valores de planicidad determinados con el Mapcheck tienden a ser inferiores que los obtenidos con el CRS. Esto es debido a que al no ser susceptibles al efecto de dosis media volumétrica, se obtienen resultados reales en zonas de alto gradiente; es decir, la abrupta caída en perfiles de campo medidos con cámaras de ionización da un estimado más negativo de la planicidad que no necesariamente es concordante con la realidad. Nótese que la diferencia entre los métodos de medición se hace menos marcada con tamaños de campo superiores a $10 \times 10 \mathrm{~cm}^{2}$, como consecuencia de la disminución en la densidad de diodos más allá del área central del arreglo.

En la comparación de los valores de simetría no se evidenció una tendencia en función del tamaño de campo o entre los métodos de medición, pero sí se pudo observar que la diferencia porcentual absoluta fue inferior al $2.0 \%$ para todas las energías en estudio.

En el estudio de comparación de fluencias se obtuvieron diferencias importantes entre las fluencias exportadas por el sistema de planificación contra las medidas con el Mapcheck para la energía de $12 \mathrm{MeV}$. Para esta energía, según se muestra en el cuadro 6, la diferencia entre la dosis de comisionamiento de TPS y la dosis medida en agua para la calibración de dosis absoluta del Mapcheck, fue de $1.4 \%$. Este incremento en la dosis medida respecto a la dosis exportada por el sistema de planificación, se reflejó claramente en los perfiles medidos con el Mapcheck (ver figura 2), lo que demuestra la sensibilidad de los diodos a variaciones en la dosis de tratamiento respecto a la dosis planificada.

En la comparación de dosis en el eje central se tienen valores ligeramente aumentados para los campos de $6 \times 6$ $\mathrm{cm}^{2}$ con energías de 12 y $16 \mathrm{MeV}$, que podrían deberse, aunado a lo dicho en el párrafo anterior, a una determi- 


\begin{tabular}{cccc}
\hline Energía $(\mathrm{MeV})$ & Planificada (B) & CAX Calibración dosis absoluta Mapcheck $(\mathrm{C})$ & $\%$ de diferencia $(\mathrm{C}-\mathrm{B}) / \mathrm{C}$ \\
9 & 97.7 & 98.2 & 0.5 \\
12 & 100.0 & 101.4 & 1.4 \\
16 & 100.1 & 99.6 & -0.5 \\
\hline
\end{tabular}

Cuadro 6: Comparación de la dosis en el eje central (CAX) del comisionamiento del sistema de planificación contra la medida con cámara de ionización para la calibración de dosis absoluta del Mapcheck

\begin{tabular}{lcccc}
\hline Energía $(\mathrm{MeV})$ & Campo $\left(\mathrm{cm}^{2}\right)$ & CAX Mapcheck A $(\mathrm{Gy})$ & CAX planificada B $(\mathrm{Gy})$ & \% de diferencia $(\mathrm{A}-\mathrm{B}) / \mathrm{A}$ \\
\hline 9 & $6 \times 6$ & 95.9 & 96.2 & -0.3 \\
& $10 \times 10$ & 98.7 & 97.7 & 1.0 \\
& $15 \times 15$ & 91.2 & 91.2 & 0.0 \\
12 & $6 \times 6$ & 96.8 & 93.8 & 3.2 \\
& $10 \times 10$ & 100.8 & 100.0 & 0.8 \\
& $15 \times 15$ & 100.5 & 99.8 & 0.7 \\
16 & $6 \times 6$ & 100.1 & 97.3 & 2.8 \\
& $10 \times 10$ & 101.3 & 100.1 & 1.2 \\
& $15 \times 15$ & 95.6 & 96.9 & 1.3 \\
\hline
\end{tabular}

Cuadro 7: Comparación de la dosis en el eje central (CAX) para el Mapcheck contra la dosis planificada para las distintas energías y tamaños de campo.

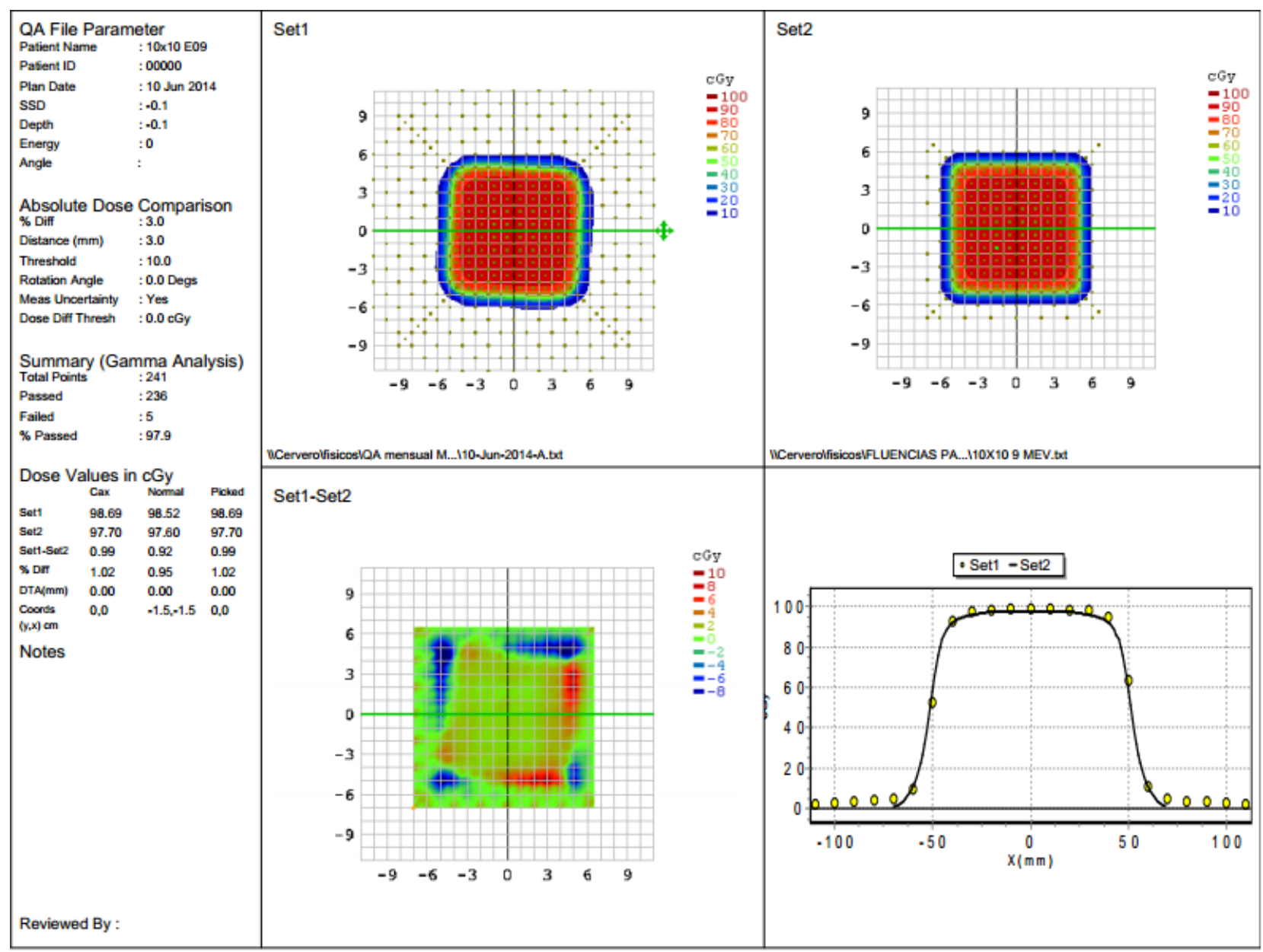

Figura 1: Documento generado con el Mapcheck para comparación de planes medidos contra planificados, para un campo de $10 \times 10 \mathrm{~cm}^{2}$ energía de $9 \mathrm{MeV}$.

nación errónea de los factores de campo.

En lo que respecta a la visualización de la distribución de dosis, es más que evidente que la pantalla del software de Mapcheck permite tener una información más completa de la distribución de dosis que con escaneo en fantoma de 


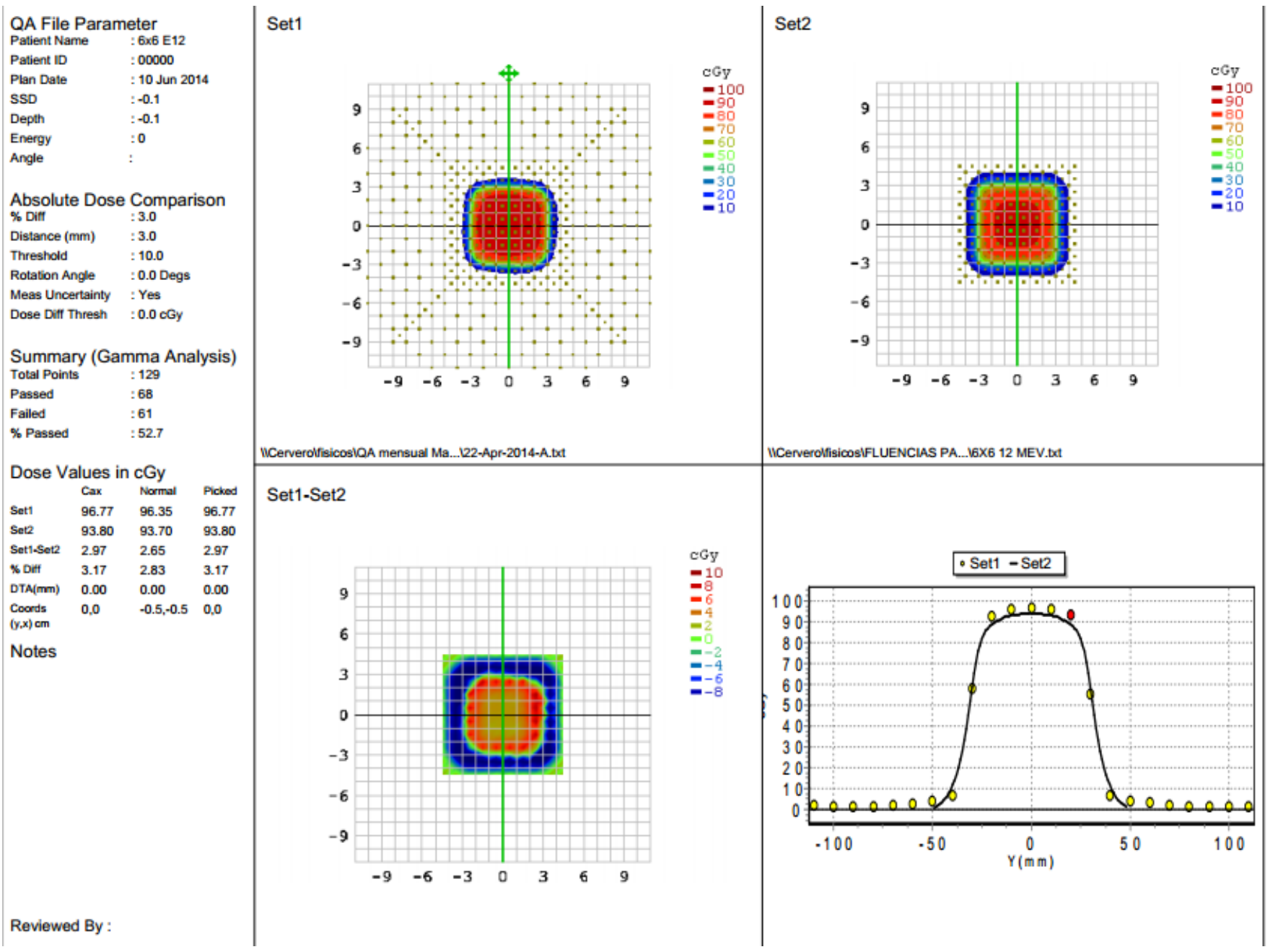

Figura 2: Similar a la Figura 1, pero para un campo de $6 \times 6 \mathrm{~cm}^{2}$, energía de $12 \mathrm{MeV}$. En este caso, el bajo porcentaje de concordancia obtenido entre los puntos y la alta dosis medida en el eje central (52.7 \% y $3.17 \%$, respectivamente), se reflejan en la diferencia entre las curvas de los perfiles para el set medido contra el planificado.

agua, en el cual únicamente se tiene información en los ejes X e Y o diagonales. El arreglo de diodos permite ver zonas frías y calientes, las cuales pueden ser indicadores de deformaciones en los conos aplicadores o funcionar como indicador visual de errores en la dosimetría clínica, producto de algún error no detectado en el comisionamiento, de ajustes en la calibración del equipo que no hayan sido debidamente incorporados en el sistema de planificación o de inexactitud del sistema de planificación en el modelaje del haz de electrones.

En el estudio se tuvieron tres limitantes importantes. La primera fue que el software de análisis del Mapcheck delimita el análisis de los datos de planicidad y simetría a campos de $5 \times 5 \mathrm{~cm}^{2}, 10 \times 10 \mathrm{~cm}^{2}$ y $15 \times 15 \mathrm{~cm}^{2}$ únicamente. Por esta razón, carece de utilidad para evaluar planicidad y simetría en campos de $6 \times 6 \mathrm{~cm}^{2}$ (incluidos en este estudio).

La segunda limitante fue el área de detección del Mapcheck, que no permite mediciones para campos superiores a $20 \times 20 \mathrm{~cm}^{2}$, dada su área máxima de $20 \times 20 \mathrm{~cm}^{2}$ del detector, y finalmente, el número limitado de tamaños de conos de electrones con tamaño inferior a $20 \times 20 \mathrm{~cm}^{2}$.

\section{Conclusiones}

El método para dosimetría de electrones presentado en este estudio presenta la ventaja de que su implementación es poco compleja y la preparación del equipo y tiempo de medición requieren mucho menos tiempo que las mediciones tradicionales en agua con fantoma de acrílico.

La concordancia entre los datos obtenidos con cámara de ionización y el arreglo de diodos, permite sumar información a los estudios previos de otros autores que indican que el uso del Mapcheck es un método confiable para evaluación de dosis absoluta en el eje central y para determinación de planicidad y simetría. Fue posible observar que el método es sensible a cambios en dosimetría absoluta, y que puede visualizarse la distribución de dosis en el plano y en los ejes X e Y, brindando más información de la distribución de dosis que otros métodos que únicamente dan información en el eje X, Y o diagonal. Presenta además, una buena reproducibilidad a corto plazo. En la evaluación del sistema de planificación, el arreglo de 


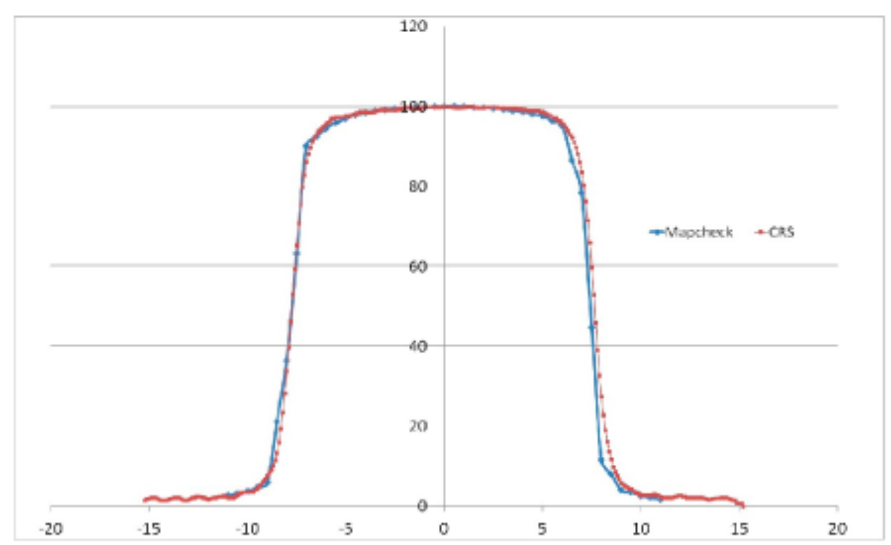

(a)

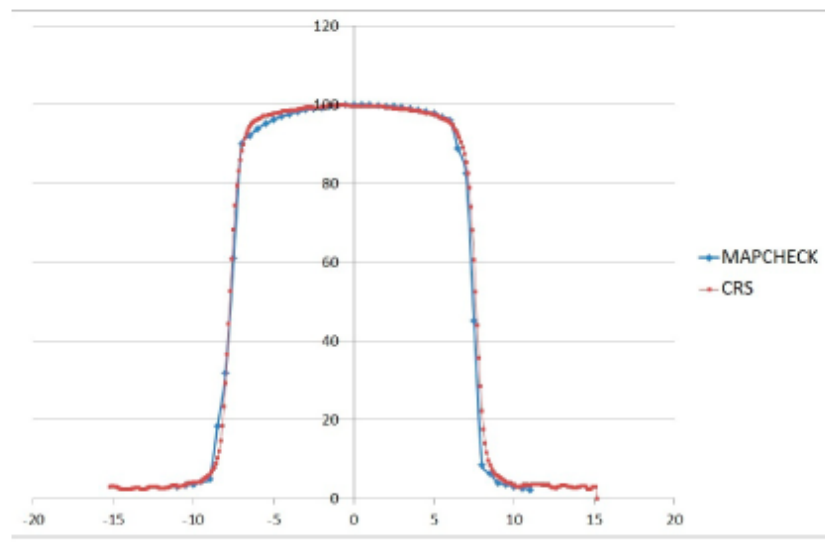

(b)

Figura 3: (a) Gráfico de perfiles de dosis para campo de $15 \times 15 \mathrm{~cm}^{2}$, energía de $9 \mathrm{MeV}$ en el exe "Y"longitudinal. (b) Gráfico de perfiles de dosis para campo de $15 \times 15 \mathrm{~cm}^{2}$, energía de $12 \mathrm{MeV}$ en el exe "Y"longitudinal

diodos nos permitió detectar divergencias entre la dosis que entrega el equipo y la calculada por el sistema de planificación.

Finalmente, se ha evidenciado la importancia de tener métodos de verificación independientes para dosimetría clínica; de lo contrario, se pueden enmascarar errores importantes en la dosimetría.

\section{REFERENCIAS}

[1] O. C. A. Gutiérrez. Diode arrays and qa of advanced techniques. Iopscience, 2010.

[2] AAPM. Physical aspects of quality assurance in radiation therapy, 1994.

[3] D. S. B. Ahluwalia. Imrt qa with 2- dimensional diode array of detectors, 2004.

[4] M. G. D. Letorneau. Evaluation of a 2d diode array for imrt quality assurance. Radiotherapy and Oncology, 70(1):199-206, 2004.

[5] C. W. e. a. F. Guo. Feasibility of using a 2d diode array system for clinical electron beam measurements. Medical Physics, 33(6), 2006.

[6] H. S. e. a. H. Khoury. Evaluation of commercial silicon diode for electron dosimetry. Nuclear instruments and methods in physics research, pages 537-539, 2007.

[7] IAEA. Absorbed dose determination in photon and electron beams: An international code of practice. Vienna, 1987.
[8] IAEA. Determinación de la dosis absorbida en radioterapia con haces externos. IAEA, 2005.

[9] P.-C. I.Torres, J. Dosimetría de haces pequeños de elelectrones mediante un método sencillo basado en película radiográfica. Revista de física médica, 5(2):90-93, 2004.

[10] B. N. P. Jursinic. A 2d diode array and analysis software for verification of intensity modulated radiation therapy delivery. Medical Physics, 30(5):870-879, 2003.

[11] P.Edimo. Clinical evaluation of fast electron monte carlo dose calculation algorithms for treatment planning systems, 2012.

[12] SunNuclearCorporation. Mapcheck software overview, 2013.

[13] SunNuclearCorporation. Sunpoint diode detectors, 2013.

[14] R. Watts. Evaluation of a diode detector array for use as a linear accelerator. Medical Physics, 2(25):247$250,1998$.

[15] P. D. e. a. Y. Ravinda. Clinical experience with routine diode dosimetry for electron beam radiotherapy. Int. J. Radiaton Oncology Biol. Phys, 48(4):1259 1265, 2000.

[16] R. d. Z. Chen. Principles and requirements of external beam dosimetry. Radiation Measurements, 41(1):S2-S21, 2007. 